\title{
THE DESCRIPTION OF HIGH CURRENT BEAM DYNAMICS USING LIE ALGEBRAIC METHODS
}

\author{
A.I.Borodich, A.A.Khrutchinsky, V.I.Stolyarsky \\ Institute of Nuclear Problems, Belorussian State University \\ 220050, Minsk, Belarus
}

\begin{abstract}
A scheme to describe a nonlaminar beam dynamics in the electromagnetic ®eld is suggested. It is based on the Lie algebraic methods and uses the quasi-equilibrium plasma model. Numerical results are represented.
\end{abstract}

\section{INTRODUCTION}

Using of the paraxial approximation for the description of high current beam dynamics leads to applying the Lie algebraic methods to calculate transfer map, that relates initial and $®$ nal coordinates and momenta of an arbitrary particle in the sixdimensional phase space [1], [2]. The main dif®culty, when dealing with a bright beam, is to take into account the in-uence of space charge on variation of beam characteristics. For laminar beams it was done in [3], [4] in terms of charged particle optics. In this paper the powerful methods, mentioned above, are used to describe high current (nonlaminar) beam dynamics, when space charge is altering. A beam is considered as a statistical system of charged particles. The results of numerical simulations for a beam being focused by longitudinal magnetic @eld are given as a simple example and compared with known facts from plasma physics.

\section{BACKGROUND MATERIAL: COMPUTATION OF TRANSFER MAP}

The location of any particle of a beam in six-dimensional phase-space is characterized by the vector $\xi\left(X X, P \quad X, P_{Y}, P_{T}\right)$. An independent variable $z$ is the coordinate along the reference trajectory. Variables $X$ and $Y$ denote transverse displacement of an arbitrary particle from the reference one, $T \bowtie x e s$ the difference of their time coordinates. And the least three variables are correspondingly the canonical conjugate momenta. Using of the reference trajectory means the description of a beam dynamics in paraxial approximation.

According to the de $®$ nition, $\operatorname{transfer} \operatorname{map} M$ relates initial and ®nal generalized coordinates and momenta of an arbitrary particle,

$$
\xi(z)=M \xi^{i n}(z)
$$

The action of this operator can be considered as a canonical transformation. Taking into account the canonical transformations generate a symplectic group, we can factorize $M$, i.e. write it as an inßnite product of Lie transformations of Lie operators associated with some homogeneous polynomials $f_{m}$ [1], [2]. Since, for the @rst, the fundamental Poisson bracket de®ning Lie operators is invariant in canonical transformations, for the second, ten symplectic group generators realize the presentation of Lie algebra, it is possible to express $f_{m}$ over polynomials $H_{m}$, obtained as one-particle Hamiltonian decomposition.
Particle's Hamiltonian is:

$$
\begin{array}{r}
H\left(X X, P \quad{ }_{X}, P_{Y}, P_{T} ; z\right)=-\frac{1}{c}\left\{\left(P_{T}+p_{t}^{0}+q \phi\right)^{2}\right. \\
\left.-\left(P_{X}-q A_{X}\right)^{2} c^{2}-\left(P_{Y}-q A_{Y}\right)^{2} c^{2}-m^{2} c^{4}\right\}^{\frac{1}{2}} \\
-q A_{z}-\frac{P_{T}+p_{t}^{0}}{v^{0}}
\end{array}
$$

( $m$ and $q$ are the particle rest mass and charge, $c Đ$ speed of light; reference trajectory parameters are marked by subscript 0 ).

Scalar and vector potentials of the electromagnetic ${ }^{\circledR}$ eld $\phi$ and A consist of two parts, that describe the in-uence of the external (Reld and space charge upon a particle:

$$
\begin{gathered}
\phi(X X, \quad ; z)=\phi^{\text {field }}(X X, \quad ; z)+\phi^{\text {beam }}(X X, \quad ; z), \\
\mathbf{A}(X X, \quad ; z)=\mathbf{A}^{\text {field }}(X X, \quad ; z)+\mathbf{A}^{\text {beam }}(X X, \quad ; z) .
\end{gathered}
$$

It is assumed $\phi^{\text {field }}$ and $\mathbf{A}^{\text {field }}$ are known. Their structure is determined by given focusing systems that are used to form or to transport a beam. If the form of $\phi^{\text {beam }}$ and $\mathbf{A}^{\text {beam }}$ is also known and the decomposition on the polynomials have been done for them, we can apply the Dragt method [1], [2] to express polynomials $f_{m}$ over $H_{m}$ and write down transfer map $M$ in obvious form. To ${ }^{\circledR}$ nd out $M$, for example, through the fourth order, the system of three matrix differential equations must be derived (their structure is adduced in [1], [2]).

\section{SPACE CHARGE CALCULATION}

Inasmuch mapping has been done for any particle of a beam as we will use one-particle distribution function $g(\xi, z)$ to calculate the potentials $\phi^{\text {beam }}$ and $\mathbf{A}^{\text {beam }}$.

The Coulomb interaction dominates in comparison with mutual collisions between the particles in high current beam [5] and we can be satis®ed by zero approximation for gas parameter $\eta$ (ratio of average potential energy to the mean kinetic) to calculate an alteration of $g(\xi, z)$ according to the Liouville theorem:

$$
\frac{d}{d z} g(\xi, z)=0
$$

Now, instead of solving the Landau-Vlasov equation and the system of the Maxwell equations jointly, we choose another way. Space charge structure proves to be invariable along $z$ within some length $l$, that is greater than the Debye radius, but don't ex ceed the range of a particle without collisions. Therefore, on every elementary length $l$ of the interaction channel time-independent space charge acts to any particle of a beam. Moreover, the distribution function satis®es the Liouville theorem (3). Hence, moving high current beam as a statistical system passes through the consequence of equilibrium states only. 
It is obviously, the volume occupied by the particles is slowly varying parameter for given adiabatic process. Thus, $g(\xi, z)$ of the quasi-equilibrium system under review assumes to obey the Boltzmann \pm Maxwell statistics along the whole interaction channel. Only the shape of the distribution function is altering on every length $l$.

To calculate $A^{\text {beam }}$ on given elementary length the quasi-stationary approximation $[6]$ can be used:

$$
\begin{array}{r}
A_{X}^{\text {beam }}(\mathrm{XX}, \quad)=0, A_{Y}^{\text {beam }}(\mathrm{XX}, \quad)=0, \\
A_{z}^{\text {beam }}(\mathrm{X}, \quad)=\frac{v_{0}}{c^{2}} \phi^{\text {beam }}(\mathrm{XX}, \quad) .
\end{array}
$$

The electric part of space charge assumes to be only transverse in respect to the reference trajectory, $\mathbf{E}^{\text {beam }}=-\operatorname{grad}\left(\phi^{\text {beam }}\right)$; the magnetic one $\mathbf{B}^{\text {beam }}=\operatorname{rot}\left(\mathbf{A}^{\text {beam }}\right) \pm$ azimuthal.

If particles are distributed according to the Boltzmann \pm Maxwell statistics and the decomposition of $\phi^{\text {beam }}$ is limited by the polynomials of the second degree, charge density of a beam $\rho(X X, \quad)$ has the Gaussian shape on every elementary length $l$. It's known that particles of a beam aren't in equilibrium, but using of the Gaussian distribution is often suitable for its description [5]. And, as it will be shown below, such simple astep by step $^{\circ}$ scheme to calculate space charge gives positive results.

The magnitude of $\phi^{\text {beam }}$ on given elementary length at some point $\left(x_{0}, y_{0}\right)$ of the beam cross-section is calculated with the help of the Green function as a solution of the two-dimensional Dirichlet problem for the Poisson equation:

$$
\begin{gathered}
\phi^{\text {beam }}\left(x_{0}, y_{0}\right)=\iint d x d y \frac{\rho(x, y)}{\epsilon_{0}} \\
\times \frac{1}{2 \pi} \ln \frac{1}{\sqrt{\left(x-x_{0}\right)^{2}+\left(y-y_{0}\right)^{2}}},
\end{gathered}
$$

where, $\epsilon_{0}$ is a dielectric permittivity of free space.

\section{ALGORITHM TO DESCRIBE A BRIGHT BEAM DYNAMICS}

So, the general scheme to describe a bright beam dynamics in external ®eld is the following. The initial cross-section of a beam is being represented as a set of the test point particles. Their transverse coordinates and momenta satisfy the Boltzmann \pm Maxwell distribution. Time coordinates are the same for all test particles and for the reference one. The energy of an arbitrary test particle differ from the reference particle energy on the quantity

$$
\begin{array}{r}
-P_{T}^{i}=c \sqrt{\left(p_{z}^{0}\right)^{2}+\left(P_{X}^{i}\right)^{2}+\left(P_{Y}^{i}\right)^{2}+m^{2} c^{2}} \\
-c \sqrt{\left(p_{z}^{0}\right)^{2}+m^{2} c^{2}} .
\end{array}
$$

According to (5) we ${ }^{\circledR}$ nd numerically values of $\phi^{\text {beam }}$ at the knots of a spatial net, that cover the cross-section of a beam, computing the two-dimensional integral by the Gauss method. Then we approximate $\phi^{\text {beam }}(\mathbb{X}, \quad)$ and $\mathbf{A}^{\text {beam }}(X X, \quad)$ from (4) by the homogeneous polynomials of the fourth degree, substitute them into the Hamiltonian (1) and receive expressions for $H_{m}$. Solving numerically the system of three matrix differential equations by the Runge-Kutta-Merson method, we determine transfer map $M$ in obvious form for every test particle. These operators are used, we ${ }^{\circledR}$ nd coordinates and momenta of the test particles at the end of the $® r s t$ elementary length. Computing the mean values and dispersions of the transverse coordinates and momenta, energy spectrum, we obtain the initial data to execute test particles on the next elementary length of the interaction channel. Repeating the procedure for calculating space charge potentials and transfer maps we carry out computations on the following steps.

The scheme described above is an algorithm for computer code TRLIE. This code calculates a bright beam characteristics when it is passing through a set of magnetic focusing elements. Veri®cation of this code was carried out by comparison with the results of the tests having executed by codes TRANSPORT (without space charge effects) and CHARLIE4F (with space charge effects) [4].

\section{EXAMPLE: HIGH CURRENT BEAM IN MAGNETIC FIELD}

Let's consider as a simple example the results given by TRLIE code, when the beam is placed at the homogeneous longitudinal magnetic ${ }^{\circledR} e l d$. They were obtained for the beam with current $1 \mathrm{kA}$, initial radius $2,5 \mathrm{~cm}$, initial divergence of the transverse momenta of $1 \%$ from the reference particle momentum at the magnetic $®$ eld induction magnitude $100 \mathrm{Gs}$ for the different kinetic energy values of the beam.

On $\circledR_{g} .1$ the beam radius evolution is shown. Obtained oscillations of a beam boundary can be identify with the surface waves in plasma with the parameter of inhomogeneity $r_{0}$, that is restrained by the external longitudinal magnetic ${ }^{\circledR} e l d$. The dispersion equation for axial symmetrical modes [7] gives the following expression for spectrum of this long-wave oscillations (frequency $\omega$ is not exceed plasma frequency $\omega_{L}$ ):

$$
\omega^{2}=k^{2} \frac{\omega_{L}^{2} r_{0}^{2}}{2} \ln \frac{1}{k r_{0}}
$$

In the table 1 magnitudes of the wave number $k$ and the frequency $w$ of the surface wave obtained by the code and the frequency $\omega$ calculated according to (6) are represented. It is obviously, the results of simulations are in good agreement with known facts from plasma physics.

\section{CONCLUSION}

The description of a bright beam dynamics, accomplished above, can be applied for any ${ }^{-}$ows. There is no restriction of an energy or current density of a beam. The validity of suggested method is limited by the paraxial approximation in sense of the accuracy to decompose one-particle Hamiltonian on the polynomials close to the reference trajectory.

\section{References}

[1] A.J. Dragt, J.M. Finn, ${ }^{a}$ Lie Series And Invariant Functions For Analitic Symplectic Maps, ${ }^{\circ}$ J. Math. Phys., vol. 17, pp. 2215-2227, 1976.

[2] A.J. Dragt, E. Forest, ${ }^{a}$ Computation of Nonlinear Behavior of Hamiltonian System Using Lie Algebraic Methods, ${ }^{\circ} J$. Math. Phys., vol. 24, pp. 2734-2744, 1983. 


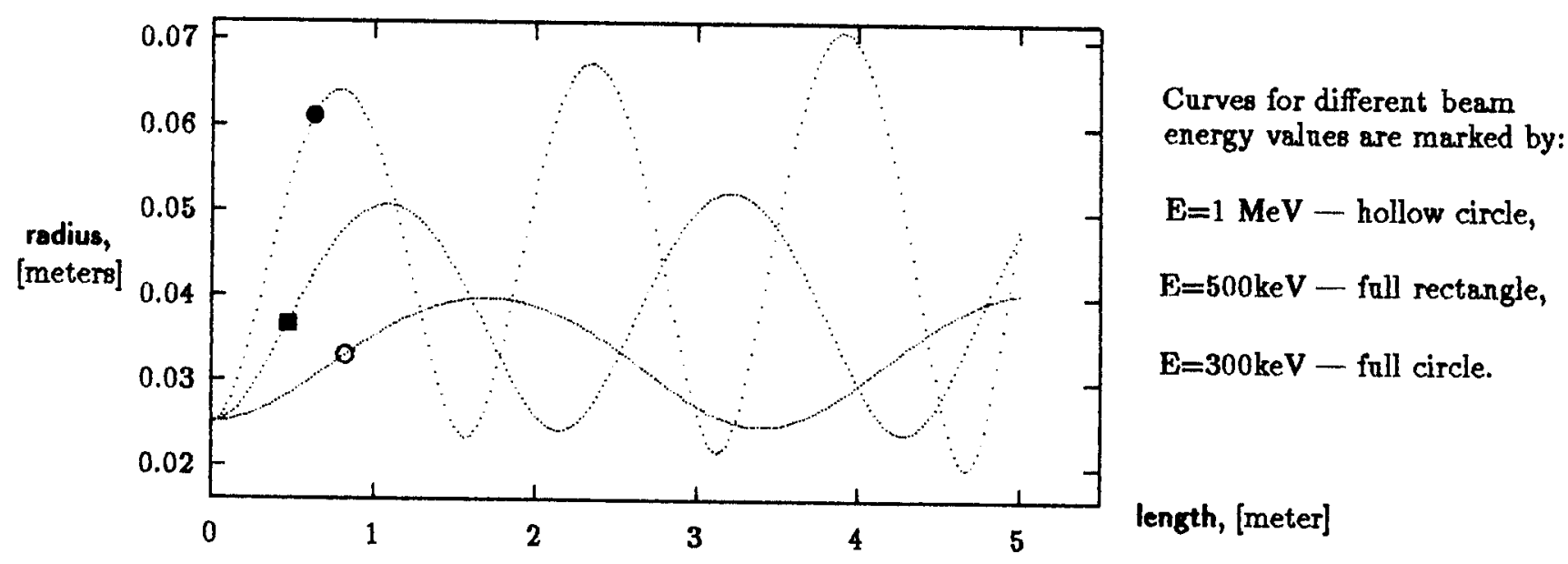

Figure. 1. Beam radius evaluation.

\begin{tabular}{|c|c|c|c|}
\hline$E, \mathrm{keV}$ & $k, \mathrm{~m}^{-1}$ & $\omega, \mathrm{sec}^{-1}$ & $w, \mathrm{sec}^{-1}$ \\
\hline 300 & 3.98 & $1.01 \times 10^{9}$ & $0.94 \times 10^{9}$ \\
500 & 2.86 & $0.81 \times 10^{9}$ & $0.76 \times 10^{9}$ \\
1000 & 1.84 & $0.57 \times 10^{9}$ & $0.53 \times 10^{9}$ \\
\hline
\end{tabular}

Table I

Characteristics of the surface waves in a beam and plasma.

[3] R.D. Ryne, A.J. Dragt, Proceedings of the 1987 IEEE Particle Accelerator Conference, vol. 2, pp. 1063-1065, 1987.

[4] R.D. Ryne, AIP Conference Proceeding, vol. 177, pp. 265274, 1988.

[5] J.D. Lawson, The Physics of Charged-Particle Beams, Clarendon Press, Oxford, 1977.

[6] I.M. Kapchinsky, Theory of Linear Resonant Accelerators, Atomizdat, Moscow, 1988.

[7] A.F. Alexandrov, L.S. Bogdankevich, A.A. Rukhadze, Fundamentals of Plasma Electrodynamics, Nauka, Moscow, 1988. 